\title{
Prosthetic joint infection after total hip arthroplasty caused by Lactobacillus paracasei
}

\author{
Charlie Tan MD, James L. Howard MD MSc, Lise Bondy MD
}

Cite as: CMAJ 2020 November 2;192:E1357-60. doi: 10.1503/cmaj.201106

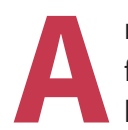

n 82-year-old woman was transferred to our hospital from another centre after sustaining a fall, which had been preceded by several days of right hip pain. She had undergone a right total hip arthroplasty (uncemented with femoral head of cobalt-chromium alloy) for osteoarthritis decades earlier. Her medical history was remarkable for left renal cell carcinoma treated with nephrectomy 16 years earlier, asthma, hypertension, dyslipidemia and hypothyroidism. The patient had no systemic illness or trauma before her symptoms.

Initial right hip radiography was negative for fracture. Subsequent computed tomography showed a complex collection at the right hip prosthesis, measuring $3.5 \times 4.8 \times 11.5 \mathrm{~cm}$ in the largest dimension (Figure 1). The patient was transferred to the orthopedic surgery service at our hospital with concern for a prosthetic joint infection.

On examination, the patient was afebrile and hemodynamically stable. There was no erythema, edema or drainage around the surgical site. She had substantial pain with motion

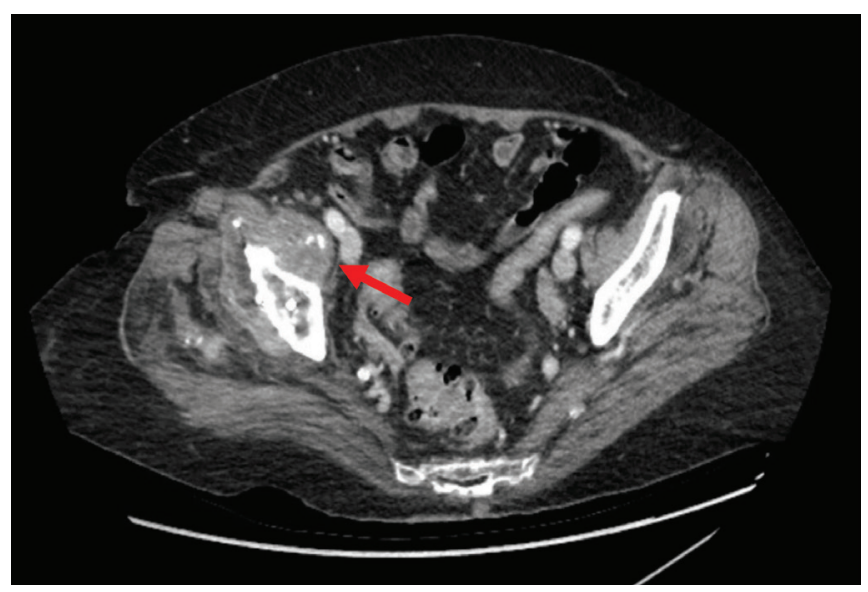

Figure 1: Computed tomography scan of the pelvis showing a complex fluid collection around the right prosthetic hip (red arrow) in an 82-yearold woman who was subsequently diagnosed with a prosthetic joint infection after right total hip arthroplasty caused by Lactobacillus paracasei. The fluid collection extended inferiorly along the femoral shaft and superiorly along the iliopsoas muscle.

\section{KEY POINTS}

- Prosthetic joint infections are serious complications of joint arthroplasties, associated with prosthetic failures, revision surgeries, and increased morbidity and mortality.

- The presence of a sinus tract to the prosthesis and 2 or more cultures positive for the same organism are considered diagnostic of a prosthetic joint infection.

- Management of prosthetic joint infections involves surgical intervention coupled with prolonged courses of antibiotics.

- Lactobacillus species are commensal organisms and are commonly used as probiotics; however, they are capable of causing severe invasive infections.

of her right hip. Investigations showed leukocytosis, with a leukocyte count of 17.3 (normal $4.5-11.0$ ) $\times 10^{9} / \mathrm{L}$, and a neutrophil count of 15.4 (normal 2.0-7.5) $\times 10^{9} /$ L. C-reactive protein (CRP) and erythrocyte sedimentation rate (ESR) were elevated to 312.7 (normal $\leq 5.0$ ) $\mathrm{mg} / \mathrm{L}$ and 101 (normal $\leq 20$ ) $\mathrm{mm} / \mathrm{h}$, respectively. Arthrocentesis showed 127 (normal < 0.2) $\times 10^{9}$ cells $/ \mathrm{L}$, $100 \%$ of which were neutrophils. Culture was positive for Lactobacillus paracasei. Two sets of blood cultures were negative. The patient was started empirically on ceftriaxone, $1 \mathrm{~g} / \mathrm{d}$ given intravenously.

A 2-stage revision arthroplasty was planned. The first stage of the 2-stage revision involves surgical removal of an infected prosthesis (or other foreign material) and implantation of an antibiotic-impregnated cement spacer, followed by 4 to 6 weeks of intravenous or highly bioavailable oral antibiotic therapy. On successful treatment of the infection, the second stage of the procedure is performed, which involves implantation of a new prosthesis.

Infected tissue and purulence were identified during the patient's first-stage revision. A cement spacer impregnated with vancomycin and tobramycin was implanted. All intraoperative samples (6/6) grew $L$. paracasei in culture. The patient was placed on a 6-week course of ampicillin, $2 \mathrm{~g}$ given intravenously every 4 hours. The patient's symptoms were quiescent during treatment with ampicillin, but the right hip pain 
recurred 2 weeks after the patient completed antibiotics. Warmth and erythema were identified on physical examination. The patient's CRP level was elevated to $164.0 \mathrm{mg} / \mathrm{L}$. Arthrocentesis showed $26 \times 10^{9}$ cells/L, $99 \%$ of which were neutrophils. Repeat first-stage revision was performed, with implantation of a new antibiotic-impregnated cement spacer. Periprosthetic purulence was identified intraoperatively. However, all tissue cultures were negative. The patient was placed on a 6 -week course of cefazolin, $2 \mathrm{~g}$ given intravenously every 8 hours.

On reassessment at the end of her antibiotic course, the patient was found to have persistent pain at her right hip. Physical examination showed erythema, serous drainage and wound dehiscence. The patient's CRP level was elevated to $67.2 \mathrm{mg} / \mathrm{L}$. She therefore underwent her third first-stage revision; all intraoperative tissue cultures were again negative. Another 6-week course of ampicillin was started. She received 3 days of adjunctive gentamicin, but this was discontinued owing to concerns of nephrotoxicity. Given the patient's progressive frailty and reasonable mobility with the cement spacer, the decision was made to leave the patient with a permanent resection arthroplasty (i.e., removal of infected hardware without reimplantation of a new prosthesis) and treat with chronic suppressive antibiotics. Ampicillin was extended for an additional 6 weeks, followed by indefinite oral amoxicillin. The patient was recovering well at her last assessment, with no substantial pain, improving right hip function and CRP level of $5.1 \mathrm{mg} / \mathrm{L}$.

\section{Discussion}

\section{Prosthetic joint infections}

Prosthetic joint infections are serious complications of joint arthroplasties, with hip and knee prostheses most frequently affected. A Canadian study found an incidence of 1.64 infections per 100 hip arthroplasties and 1.52 infections per 100 knee arthroplasties over a 3-year period. ${ }^{1}$ Risk factors include obesity, diabetes mellitus, immunosuppressive medications, previous revisions and/or infections of the prosthesis, rheumatoid arthritis and malignancy. ${ }^{2}$ Prosthetic joint infections are associated with poor outcomes, with 5-year mortality rates of greater than $20 \%$ and threefold higher age-adjusted odds of mortality compared with the general population. ${ }^{3}$

The most common mechanism of infection involves introduction of microorganisms during the initial arthroplasty; others include spread from a contiguous infection or hematogenous seeding. ${ }^{2}$ Staphylococcus aureus, coagulase-negative staphylococci and streptococci are the most frequent causative microorganisms; other important bacteria include gram-positive bacilli, such as Cutibacterium acnes, and gram-negative bacilli. ${ }^{2}$ Early-onset infections within 3 months of the initial arthroplasty are generally caused by more virulent organisms, including S. aureus and gram-negative bacilli. More indolent organisms, such as coagulase-negative staphylococci and C. acnes, comprise the majority of delayed-onset infections, which present within 1 to 2 years of implantation. Late-onset infections, occurring years after implantation, are more frequently the result of hematogenous seeding, with $S$. aureus being most common. ${ }^{2}$
The preoperative diagnosis of prosthetic joint infections is often challenging. Early-onset infections present with features of septic arthritis, including acute pain, erythema, edema, drainage and poor wound healing. Delayed-onset infections can masquerade as aseptic failure, with chronic pain and loosening. The presence of a sinus tract communicating with the prosthesis is diagnostic for infection. On laboratory investigations, CRP and ESR are frequently elevated. Diagnostic arthrocentesis should be performed in all suspected cases, preferably in consultation with an orthopedic surgeon owing to the potential for direct inoculation of bacteria. Elevated synovialfluid leukocyte count with neutrophilic predominance and positive bacterial culture are indicative of infection. Synovialfluid leukocyte counts are often much lower than what is seen in native-joint septic arthritis.

The diagnosis is often made intraoperatively. Two or more tissue cultures (or in combination with specimens from arthrocentesis) positive for the same organism is diagnostic for a prosthetic joint infection, though isolation of virulent organisms (e.g., S. aureus, gram-negative bacilli) in a single specimen is highly concerning as well. Purulence surrounding the prosthesis and histopathologic evidence of inflammation are also suggestive. Given the importance of microbiologic identification, antimicrobials should be withheld for at least 2 weeks before specimen collection in clinically stable patients. Guidelines are available to assist clinicians with diagnosis., ${ }^{4,5}$

The treatment of prosthetic joint infections includes both surgical and antibiotic management. We suggest consulting an expert in infectious diseases. The primary surgical options, with their indications and associated antibiotic management, are summarized in Box 1. Débridement, antibiotics and implant retention (DAIR) involves irrigation and débridement of the infected prosthesis, often with exchange of the polyethylene liner covering the joint interface. One-stage revision arthroplasty, common in Europe and increasingly used in North America, involves removal of the infected prosthesis and reimplantation of a new prosthesis during the same surgery. ${ }^{4}$ There is increasing evidence that 1-stage arthroplasties have similar outcomes to 2-stage revision arthroplasties, which have generally been considered the gold-standard method for treating prosthetic joint infections. ${ }^{6}$ The antimicrobial regimen for both DAIR and 1-stage revision arthroplasty involves up to 6 weeks of intravenous antibiotics, followed by 6 weeks of a highly bioavailable oral antibiotic (extended to 12 weeks for knee arthroplasty infections). Adjunctive rifampin is indicated with staphylococcal infections. Chronic suppressive oral antibiotics should be considered on a case-by-case basis. Given the need for prolonged oral antibiotics, these options are ideally performed when the causative organism is identified preoperatively and susceptible to highly bioavailable oral antibiotics. However, the microbiologic etiology may be elusive until intraoperative cultures are obtained. Two-stage revision arthroplasty, described in this case, should be considered only for patients who can withstand 2 separate surgeries. Permanent resection arthroplasty, which was ultimately performed in our patient, or amputation may be required in select severe or nonresolving cases. ${ }^{4}$ 
Box 1: Indications for, and associated antibiotic management of, the most common surgical options for treatment of prosthetic joint infections ${ }^{4}$

\begin{tabular}{ll} 
Surgery & \multicolumn{1}{c}{ Indications } \\
Débridement, antibiotics and implant retention & - Infection within 30 days from implantation \\
- Duration of symptoms 3 weeks & - Well-fixed prosthesis with no sinus tract \\
- Microbiologic etiology identified preoperatively and & susceptible to highly bioavailable oral antibiotics \\
1-stage revision arthroplasty & - Total hip arthroplasty \\
- No important comorbidities & - Healthy reserve of bone and soft tissue \\
- No sinus tract & - Microbiologic etiology identified preoperatively and \\
& susceptible to highly bioavailable oral antibiotics \\
- Difficult-to-treat microorganisms (e.g., MRSA, & Candida, highly resistant organisms) \\
- Poor soft tissue but adequate bone stock & - Presence of sinus tract is not a contraindication \\
- No previous 2 -stage revision arthroplasty for & infection \\
- Patient able to undergo 2 separate surgeries
\end{tabular}

Associated antibiotic management

Up to 6 weeks of intravenous antibiotics, followed by 6 weeks of highly bioavailable oral antibiotics (extended to 12 weeks for infections of knee arthroplasties)

Up to 6 weeks of intravenous antibiotics, followed by 6 weeks of highly bioavailable oral antibiotics (extended to 12 weeks for infections of knee arthroplasties)

4-6 weeks of intravenous or highly bioavailable oral antibiotics after first stage

Note: MRSA = methicillin-resistant Staphylococcus aureus

\section{Lactobacillus species}

Lactobacillus species are gram-positive nonsporulating anaerobic bacilli that are part of the normal oral, gastrointestinal and female genitourinary microbiota. Probiotic strains are widely used to improve gastrointestinal health. However, Lactobacillus can cause clinically significant infections, particularly in patients who are immunocompromised or with critical illness. ${ }^{7,8}$ Bacteremia and infective endocarditis are most common, though intra-abdominal, respiratory and neurologic infections have also been reported. ${ }^{8}$ Lactobacillus has rarely been implicated in prosthetic joint infections, with only 3 previous case reports. ${ }^{9-11}$

The source of our patient's L. paracasei prosthetic joint infection is not clear. Computed tomography of her abdomen and pelvis did not show any intra-abdominal pathology. She had not undergone recent odontogenic, abdominal or genitourinary procedures. In addition, the persistence and severity of our patient's prosthetic joint infection was unexpected, given appropriate surgical and antibiotic treatment, and the absence of substantial immunocompromise. Adding gentamicin for synergy following the initial first-stage revision may have more effectively treated the infection. Although no other microorganisms were identified, cultures may have been sterilized by perioperative antibiotics; mixed infection by other pathogens is therefore possible. Mycobacterial and fungal cultures were never ordered, but the patient had no risk factors for infections with these organisms. Invasive Lactobacillus infections have been associated with substantial mortality, though in highly comorbid patients. ${ }^{8}$

There are no guidelines on antibiotic therapy for invasive infections by Lactobacillus. Bacteremia and, by associ- ation, other invasive infections are usually treated with benzylpenicillins (e.g., penicillin G) or aminopenicillins (e.g., ampicillin), often in combination with an aminoglycoside. ${ }^{7}$ In general, studies have shown that Lactobacillus has greater susceptibility to penicillins, carbapenems, clindamycin and erythromycin..$^{7,8}$ However, susceptibilities differ by species; one study of 85 blood isolates of Lactobacillus found widely variable susceptibilities to penicillins between species. ${ }^{7}$

\section{Conclusion}

This case highlights the diagnostic and therapeutic considerations of prosthetic joint infections. Multidisciplinary management is encouraged, as treatment involves surgical intervention coupled with prolonged antibiotic regimens. Invasive infections by Lactobacillus species are rare but have been well documented; isolation of Lactobacillus therefore requires treatment in the appropriate clinical contexts.

\section{References}

1. Roth VR, Mitchell R, Vachon J, et al. Periprosthetic infection following primary hip and knee arthroplasty: the impact of limiting the postoperative surveillance period. Infect Control Hosp Epidemiol 2017;38:147-53.

2. Tande AJ, Patel R. Prosthetic joint infection. Clin Microbiol Rev 2014;27:302-45.

3. Lum ZC, Natsuhara KM, Shelton TJ, et al. Mortality during total knee periprosthetic joint infection. J Arthroplasty 2018;33:3783-8.

4. Osmon DR, Berbari EF, Berendt AR, et al. Diagnosis and management of prosthetic joint infection: clinical practice guidelines by the Infectious Diseases Society of America. Clin Infect Dis 2013;56:e1-25.

5. Parvizi J, Tan TL, Goswami K, et al. The 2018 definition of periprosthetic hip and knee infection: an evidence-based and validated criteria. J Arthroplasty 2018;33:1309-14e2.

6. Kildow BJ, Della-Valle CJ, Springer BD. Single vs 2-stage revision for the treatment of periprosthetic joint infection. J Arthroplasty 2020;35(Suppl):S24-30. 
7. Salminen MK, Rautelin H, Tynkkynen S, et al. Lactobacillus bacteremia, species identification, and antimicrobial susceptibility of 85 blood isolates. Clin Infect Dis 2006;42:e35-44.

8. Lee MR, Tsai CJ, Liang SK, et al. Clinical characteristics of bacteraemia caused by Lactobacillus spp. and antimicrobial susceptibilities of the isolates at a medical centre in Taiwan, 2000-2014. Int J Antimicrob Agents 2015;46:439-45.

9. Orkaby AR, Chen B, Iliaki EF, et al. A curious case of Lactobacillus casei in a prosthetic joint: Was it the yogurt? J Am Geriatr Soc 2012;60:1177-8.

10. Bennett DM, Shekhel T, Radelet M, et al. Isolated Lactobacillus chronic prosthetic knee infection. Orthopedics 2014;37:e83-6.

11. Somayaji R, Lynch T, Powell JN, et al. Remote transient Lactobacillus animalis bacteremia causing prosthetic hip joint infection: a case report. BMC Infect Dis 2016;16:634.

Competing interests: James Howard reports funding from Stryker, DePuy, Smith \& Nephew, Zimmer, Microport and Intellijoint, and is a stockholder in PersaFix Technologies. No other competing interests were declared.

This article has been peer reviewed.

The authors have obtained patient consent.

Affiliations: Division of Infectious Diseases (Tan), University of Toronto, Toronto, Ont.; Division of Orthopaedic Surgery (Howard), Western University; Division of Infectious Diseases (Bondy), St. Joseph's Health Care, London, Ont.

Contributors: Charlie Tan and Lise Bondy contributed to the conception and design of the work. All authors contributed to the acquisition, analysis and interpretation of data. All authors contributed to drafting the work and revising it critically for important intellectual content. All authors give final approval of the version to be published and agree to be accountable for all aspects of the work.

Correspondence to: Charlie Tan, charlie.tan@mail.utoronto.ca

The section Cases presents brief case reports that convey clear, practical lessons. Preference is given to common presentations of important rare conditions, and important unusual presentations of common problems. Articles start with a case presentation (500 words maximum), and a discussion of the underlying condition follows (1000 words maximum). Visual elements (e.g., tables of the differential diagnosis, clinical features or diagnostic approach) are encouraged. Consent from patients for publication of their story is a necessity. See information for authors at www.cmaj.ca. 